\title{
Conceptual and Instrumental Universalization and Implementation of Scheduling Algorithm of Extreme Experiment for Complex Objects
}

\author{
Oleg N. Dmitriev \\ Moscow Aviation Institute (National Research University) (MAI), Volokolamskoe Highway, 4, \\ 125993, Moscow, Russia
}

\begin{abstract}
The computer technology searching for a preferable point in any factor space delivers to the extremum with regard to quantitative scalar response within scope of intelligent DSS-sphere, which was considered in this paper. The problem of universalization and planning (design) of extreme experiments in relation to complex objects in the conditions of significant limitation including the allowable number of experiments and sub-catastrophic duration of them was solved. A technology that involves the separation of three linked stages of optimization was proposed.
\end{abstract}

Keywords - design of experiment, extreme experiment, factor-response representation, intelligent DSS, optimization of decisions.

\section{Introduction}

The justification of any management decisions provides their optimization, because this optimization is an indispensable function of management [8]. The optimization, as it is well known, can be carried out on real objects of management and their models, including natural, semi-subsistence and mathematical (analytical and simulation) ones. Natural objects can be technical, economic, social, etc.

DOI: 10.18421/TEM94-61

https://doi.org/10.18421/TEM94-61

Corresponding author: Oleg N. Dmitriev, Moscow Aviation Institute (National Research University) (MAl), Moscow, Russia.

Email: olegdmitriev@yandex.ru

Received: 26 May 2020.

Revised: 08 November 2020.

Accepted: 14 November 2020.

Published: 27 November 2020.

(c) BY-NC-ND (C) 2020 Oleg N. Dmitriev; published by UIKTEN. This work is licensed under the Creative Commons Attribution-NonCommercial-NoDerivs 4.0 License.

The article is published with Open Access at www.temjournal.com
As a rule, in the realities of modern management, it is not possible to solve the optimization problem with classical regular methods, such as checking whether the necessary and sufficient conditions for the existence of an extremum with respect to derivatives of the objective function are met, and even more so with an output to a formula or numerical value. This is primarily due to the fact that the corresponding response surfaces often have an a priori completely unknown, arbitrary appearance, various kinds of discontinuities, and also fundamentally vary from one management situation to another.

Of course, the physical conceivability and reproducibility of these experiments are very serious and often insoluble problems in the case of real objects. In fact, for example, the multiple re - entry of ocean-going ships into the water at the shipyard and the destructive experiments of the processing centers in the "hot shop" of the enterprise look like anti-scientific fantastic scenarios searching for the optimum. By the way, there are cases when computer experiments were also unrealizable or generated the phenomenon of non-reproducibility.

A fairly widespread approach to development technologies of optimization within administrative decisions consists of their development and localization for specific objects, conditions, etc. Thus, the whole fan of algorithms is developed and applied. At realization of this conceptual approach too many resources are spent and chances regarding the administrative personnel to make a technical or semantic error increase, owing to a wrong choice of technologies. As a rule, optimization provides planning and carrying out experiments on the control object or its model as reception of the analytical description of positioning of an optimum (for example, formulaic or numerical) for difficult objects, in the general case it is unrealizable.

Therefore, there is a quite natural and not quite trivial conceptual idea: to develop a universal algorithm for planning an extreme experiment, suitable for use both for full-scale and computer experiments (in some way, a multipurpose optimization algorithm). 
This algorithm can also be interpreted as a tool for extracting information about such a property of an information object as its extremum in a certain subspace of characteristics, localized by the optimization criterion and imposed restrictions.

\section{Methodology}

The general structuring of research methodology is covered quite fully, in particular, in [1], [2], [3] and in some others.

The reflected author's development is of a conceptual, methodological and instrumental nature. It mainly concerns the part of the system-technical tools of the information and consulting system within management decision support that is associated with the implementation of such a management function as optimization of management decisions.

As the theoretical basis of the author's research, the following methods were used:

- general theory of management;

- system analysis;

- optimization theory;

- probability theory;

- uncertainty theory;

- information theory;

- theory of experiment planning (designing);

- set theory

We also used some other scientific theories related to the study of operations in the field of operating with complex techno-economic objects.

\section{Results and Discussion}

\section{Forerunners and the Applicability of their Developments}

Unfortunately, the appearance and application of algorithms for planning extreme experiments in Russian science, according to external features, receive insufficient attention.

So, as on 25.05.2020, on the website of the Russian State Library, www.rsl.ru, a total of 153 publications on experiment planning for the period after 1980 some books were presented, including only one monograph. However, not a single publication was specifically devoted to the subject of planning extreme experiments, and more than $90 \%$ of publications were teaching aids for students, mainly from regional universities.

According to external signs, the picture on the website of the Library of Congress of the USA at www.loc.gov is even more depressing. There such references were not revealed at all. There are only local blotches of books on similar topics, primarily related to simulation modelling.
We see something similar in the number of publications carried out by the publishing complex IGI Global. On the site of this publishing complex (www.igi-global.com) as on 25.05.2020 there are no editions with such a direct dictionary combination, although there is a key combination for the "extreme experiment" and its variations with samples of several thousand items. It is characteristic, for example, that in some rather solid publications of the aviation field, which is the most familiar for the [14], the problems of optimal experimentation are not even marked. This applies to the production and technical exploitation.

The identification of specialized articles turned out to be quite accessible in the field of operations research by referring to the site www.pubsonline.informs.org (as of 25.05.2020). There are about 2800 positions there - [15], [16], [17], for example. A selective, representative review showed that deep works were presented there, but focused not on particularly complex information objects; including those for which ideas about the analytical representation of the response surface can be formed, i.e. indirectly identify the objective function in an analytic or semi-analytic representation. In addition, apparently there were no particularly strict restrictions on the number of experiments in the presented studies. Among other things, it was connected with the fact that in the researches available for public analysis usually "heavy" and, accordingly, especially resourceintensive software is not used, and in the part of fullscale experiments its role is limited to processing the results of their performance.

In some available sources, some local issues are discussed, but mainly in relation to the statistical processing of the results in the field and computer experiments. These gleaned components are referred to as contextual when necessary.

In fact, the only exception, at least in the USSR and in Russia, is the ancient classic edition on the theory of experimental design [7]. But the tools offered are focused on research and noncomputerized operations for the formation of management decisions.

From the well-known practice, the planning/design of experiments (the abbreviation DoE is often found), including extreme experiments (DoEE accordingly, but less often), is carried out empirically: plans are formed on the basis of the attitudes of decisionmakers and from certain considerations of analysts. Obviously, with this approach, there are schemes of the administrative-empirical game of "roulette". Sometimes, due to good intuition and generalizations of experience, they lead to an acceptable result, but in general they represent a certain ritual act, which is still better than its complete absence. 
Numerous researchers put forward considerations simply in relation to experiment planning (for example, [9]), and sometimes perform constructions to optimize the experiment (for example, [10]). A number of studies are focused on universal methods (for example, [11]), and some on object-oriented and subject-oriented ones (for example, related to industrial production [12]). Some of the experimental planning methods are intended to identify the response surface, and some are comparative (for example, [13]).

The problems of this nature have appeared long ago in all high-tech areas, including the design and development of the active part of fixed assets within industrial enterprises, the most visible component of which is technological equipment, including the machinery fleet and tools for providing technological processes for production. This problem is relevant for all stages of the life cycle of complex technical products and for mathematical models describing the parks of these products for these stages.

However, it was not possible to reveal their exhaustive or at least strongly approximated to such a scientific-design solution in the available sources.

Therefore, the problematic issue should be recognized as relevant and for some reason touched upon in publications to an extremely small extent.

The situation outlined does not give rise at all to a conclusion about the pioneering or breakthrough profile of the developments described in this article, but merely allows us to state that it was only necessary to largely follow the "tabula rasa" scheme.

\section{Selected Universal Properties of Objects}

Sophisticated technical, economical, information and other informatively complex objects have a number of properties similar in terms to the organization and implementation of extreme experiments on them, namely:

- The space of factors (optimizing factors) is, as a rule, multidimensional, i.e. the number of factors exceeds two;

- The factors can be described by several variables: quantitative and qualitative. However, these variables are always postulated as deterministic (for stochastic and uncertainty factors, as their specific implementations are deterministic). Moreover, these variables can be both continuous and discrete, although qualitative variables are most often interpreted as "discrete" variables in some way: for example, by numbering non-numeric values. A typical case is when a point in the factor space is "mixed" in discreteness: in one dimension it is discrete, and in others it is continuous. Points in the factor space can generate their finite sets, countable sets, and power sets of the continuum;

- The points in the factor space can be acceptable and unacceptable, both by the values of the factors and by the values of the derived responses. The configuration of the spaces of permissible response values can be arbitrary: in form, in connectivity, in size, determined by the power of the set, etc. However, it is obvious that when planning an extreme experiment, the inadmissibility of points of factor space by the response values cannot be considered;

- Points in the factor space may be possible for research and mandatory for research: the second case is related to the fact that they can be imputed by legal or volitional acts of management personnel;

- The composition of factors in planning an extreme experiment is a priori given and cannot be changed during the planning and conduct of an extreme experiment;

- The limiting ranges of variation of factors and the limiting discreteness of variation of factors are given a priori. They are unchanged from one experiment to another;

- The response space is one-dimensional and has a quantitative character, and the corresponding response, as a rule, is described by a continuous deterministic quantity or is intermediately reduced to a set with a finite number of elements: stochastic realizations. This is explained by the fact that in optimizing management decisions, scalarization of the vector optimization criterion is inevitably performed and is determined by applying statistics (most often initial moments and quantilities). The conclusion according to the deterministic result allows defining uncertainties in response to implementations. The latter problem is characteristic, first of all, for experiments with the phenomenon of the origin of trajectory bifurcations. In this case, the procedures for resolving uncertainties should be applied and some others. The problem of scalarization of optimization criteria for management decisions is external to the problem under consideration [8] and some others.

\section{Basic Requirements for the Algorithm}

We can formulate the requirements to the synthesized algorithm of extreme experiment planning:

- accounting for the above properties as features;

- invariance with respect to the assigned composition of factors and content filling of the response; 
- providing an arbitrary plan ability which need to be resolved;

- providing the best convergence as much as possible in the field of the global optimum;

- accounting for a priori strong limitations of the permissible total number of experiments;

- "automaticity" of the experiment, implying that the experimenter is not obliged to intervene in the course of its implementation;

- possibility of introducing a "start-stop" mode, when the execution of the algorithm can completely or temporarily interrupt the pause initiated by the experimenter. This is due to the following: first, in experiments on field and semi-natural objects, experiments are most often carried out in the form of a series of spaced apart times; secondly, experiments on software implementations of models can last for a long time; and third, the abandonment of computer systems in the "deserted" version of operation is dangerous;

- connectivity with software implementations of models, if the object is a mathematical model;

- ensuring the receipt of an empirical estimate of the remoteness of the received rational management decision from the unknown optimal;

- software feasibility and applicability of software implementation on computing equipment with a non-unique configuration for modern Russia.

\section{Decomposition of the Algorithm}

Correspondingly, a decision was made considering the research in the field of factor planning, random planning, etc. [6], [7] to form the considered algorithm as a three-stage that includes the following procedural steps [8]:

- steep ascent;

- investigation of almost stationary area;

- evaluation of the remoteness of the obtained rational management decision by comparison with the unknown optimal one.

Due to the fact that the number of experiments is essentially limited, the rotatability of planning, which is desirable from the point of view of the uncertainty of the location of the desired optimum, as well as $D$ optimality, is fundamentally impossible to achieve if we use classical fractional replicas at the first stage and the Rotatable Central Composite plan (RCC plan) at the second.

\section{Multiple Comparison Method (MCM) for Matching Points}

To select the preferred ones of the compared points in the factor space, a procedure was proposed for comparing the corresponding responses. In this case, there is an assumption, which does not break the generality (the signature method of inverting the type of extremum is well known) and makes the maximization of the quantitative response $R$ reasonable.

When comparing the points of the factor space, we compare the selected average realizations of the responses $M^{*}\{\hat{R}\}$ obtained during the experiment at the object in these points.

It has been established that the comparison of sample averages without evaluation of the significance of the difference between them leads to unacceptable levels of probability of comparison errors (such probability, as it has been established, exceeds the value of 0.2 ). Therefore, the choice was made in favor of MCM-procedure based on Bonferroni's $t$-statisticians, which are redundant in relation to violations of the normality of the law regarding distribution of the sample average response and the heterogeneity of its dispersion [4], [5]. Their advantage is also their ability to work in experiments without duplication. The only limitation due to the choice of method is uniform duplication of experiments. In contrast to the procedure described in [4], [5], the reference level is not the level of error for comparison, but the specific level of significance, because the Bonferroni's estimation at a large number of compared points is excessively conservative. The use of multiple ranking methods [6] is unacceptable due to the limited number of experiments.

The selected MCM method consists of a sequential analysis of samples comprising the response values of the species:

$$
\begin{aligned}
& \left\{\hat{R}_{11}, \hat{R}_{12}, \ldots, \hat{R}_{1 d}\right\}, \ldots \\
& \ldots,\left\{\hat{R}_{v 1}, \hat{R}_{v 2}, \ldots, \hat{R}_{v \mu}, \ldots, \hat{R}_{v d}\right\}, \ldots, \\
& \ldots,\left\{\hat{R}_{N_{c . p .}, \ldots}, \ldots, \hat{R}_{N_{c . p .}, d}\right\}
\end{aligned}
$$

where $N_{c . p .}$ is the number of points of factor space to be compared;

$d$ is the number of repeated implementations of the experiment with the object (corresponds to $d-1$-th multiple duplication of experiments in the compared points); 
$\hat{R}_{\nu \mu}$ is the $\mu$-th implementation of response in the point of factor space number $v$.

On the iteration number $u$ from $N_{c . p .}$ of this analysis step by step we can check the implementation of the set of conditions (obviously, for $d>1$, otherwise there is no experiment):

$$
\left\{\begin{array}{l}
M_{\text {now }}^{*}\{\hat{R}\}>M_{\text {past }}^{*}\{\hat{R}\} ; \\
0 \text { (here - symbol zero) does not belong to the range } \\
M_{\text {now }}^{*}\{\hat{R}\}-M_{\text {past }}^{*}\{\hat{R}\} \pm t_{2 d-2}^{\alpha}\left(\sigma_{\text {past }}^{*^{2}}\{\hat{R}\}-\right. \\
\left.+\sigma_{\text {now }}^{*^{2}}\{\hat{R}\}\right)^{1 / 2} d^{-1 / 2}
\end{array}\right.
$$

and the current sample standard deviation is determined by the formula:

$$
\left\{\begin{array}{l}
\left.\sigma_{\text {now }}^{*^{2}}\{\hat{R}\}\right)=(d-1)^{-1} \sum_{j-1}^{d}\left(\hat{R}_{u j}-M_{\text {now }}^{*}\{\hat{R}\}\right)^{2}, \\
\text { if } d>1 \\
\sigma_{\text {now }}^{*^{2}}\{\hat{R}\}=0 \\
\text { if } d=1
\end{array}\right.
$$

$t_{2 d-2}^{\alpha}$ is $\alpha$-quantile of $t$-distribution with $2 d-2$ freedom degrees;

$M_{\text {past }}^{*}\{\hat{R}\}$ is significantly better sample average, which was found on the iteration $u-1$ (for $u=1$ the value is actually a non-existent experiment $M_{\text {past }}^{*}\{\hat{R}\}=0$, that is, for this iteration, the preceding value is conditional);

$\sigma_{\text {past }}^{*^{2}}\{\hat{R}\}$ is sample standard deviation of the best sample means, which was found on the iteration number $u-1$ (for $u-1$ the value $\sigma_{\text {past }}^{* 2}\{\hat{R}\}=0$, that is, for this iteration, the preceding value is conditional).

It is clear that the roots of even degrees $(2,4$, etc.) everywhere are taken as positive values. So, there are no signs of module expressions.

If this complex condition is satisfied, the new significantly better mean is considered to be the value of $M_{\text {now }}^{*}\{\hat{R}\}$. Otherwise, the value $M_{\text {past }}^{*}\{\hat{R}\}$ is kept and use don the iteration number $u+1$.

The quantile value $t_{2 d-2}^{\alpha}$ is estimated numerically: by iteratively comparing the estimate of the integral of the corresponding standard distribution density with the value $\alpha$.

This estimation is made by consecutive comparison of an estimation of an integral $I_{\text {est. }}\left(x_{\{\text {index\} }}\right)$ with the threshold value $\alpha$.
It is accepted that:

$$
t_{2 d-2}^{\alpha}=x_{\rho},
$$

if on the iteration $\rho+1$ this if the following:

$$
\left\{\begin{array}{l}
I_{\text {est. }}\left(x_{\rho}\right)>1-\alpha ; \\
I_{\text {est. }}\left(x_{\rho+1}\right)<1-\alpha .
\end{array}\right.
$$

Let us now consider how the selected stages of the proposed algorithm are implemented.

\section{Steep Ascent procedure}

This procedure involves constructing a Linear Regression Model (LRM) of the response surface and moving along it in the direction of the desired optimum.

It can be implemented or skipped. Significant problems arise in the case of qualitative factors, because the linearity of the regression model according to the conditional numbers of gradations of qualitative factors, even in terms of strict interpretation, causes many problems. In addition, the extremum search operator can assume that the initial point of the experiment is close enough to the desired optimum.

The general block diagram of the implemented steep ascent algorithm is shown in Figure 1.

Now we can consider how its components are implemented.

To minimize the number of experiments which are to be carried out with the object and maintain the specified resolution ability of the plan $D$ at construction of LRM the response surface was set and solved the problem of optimizing the fractional replication of plan - value $\boldsymbol{p}$ for plans like $2_{D}^{K-p}$ of the following type:

$$
p \rightarrow \max
$$

under the upper bound constraint of the value of the fractional replication $p$, which comes from the given values of the number of factors $K$, resolution ability of plan $D$ and also from the standard procedure for obtaining generating ratios: 


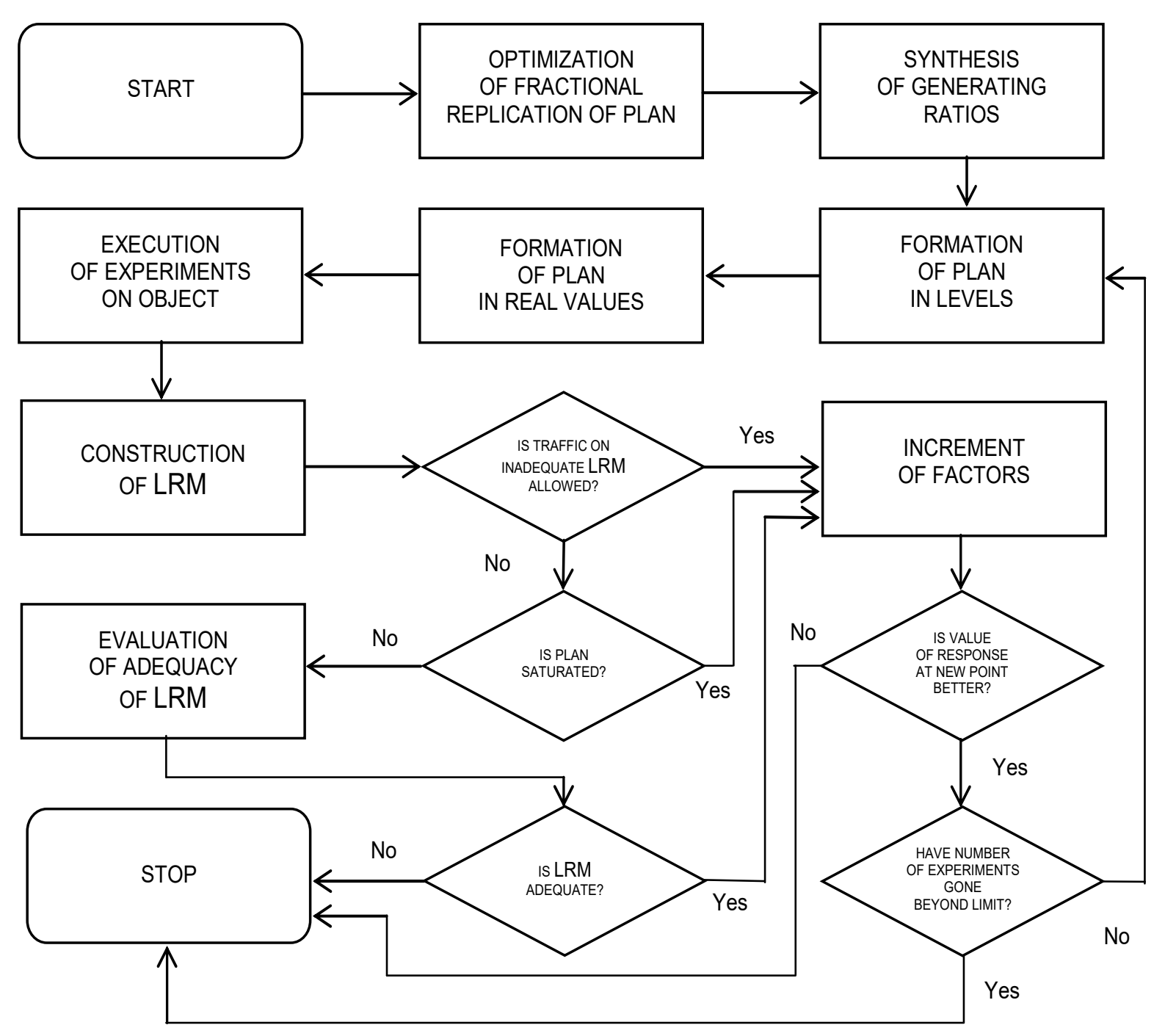

Figure 1. General block diagram of the constructed algorithm of steep ascent

$$
\sum_{n=D-1}^{K-p} C_{K-p}^{n} \geq p
$$

Here $C_{*}^{* *}$ is the standard expression for combinatorics "from ... to ...".

It should be noted that in most of the known studies the problem of optimizing the fractional replication of the plan was not posed and was not solved respectively.

To find the optimal fractional value of the replication $p_{\text {opt. }}$ the method of sequential search of values is used, $p$ from the values in the direction of reducing (algorithmic variable $\eta$ ). Once the limit is reached, the optimal value of the replication's fractional value $p_{\text {opt. }}$ is accepted equal to this boundary value on $p$. Values $\eta !,(K-p) !$ and $(K-p-\eta) !$ are respectively as values $\Gamma(\eta-1)$,
$\Gamma(K-p-1)$ and $\Gamma(K-p-\eta-1)$, where $\Gamma(x)$ is well-known $\Gamma$-function of the argument $x$. The calculation of $\Gamma$-function is performed using the wellknown infinite Weierstrass's bundle. The required accuracy is provided if at least 10 factors are used in the Weierstrass's bundle. The time to find the optimal fraction of a replica $p_{\text {opt. }}$ depends on the number of factors $K$ (approximately $0.05 K$ ).

In the course of the research, including the optimal values of the fractional replications were found $p_{\text {opt. }}$ for the volumes $K \in \overline{1,40} ; D \in \overline{\mathrm{III}, \mathrm{VIII}}$, where we can find the number of experiments for the plan like $2_{D}^{K-p_{\text {opt. }}} \quad$ [8] and exceeding them over the number of experiments in the deep plan. As we can see for example in the calculating results [8], when $D=$ III and $K \leq 15$, there is no significant gain in the number of experiments in the transition to saturated 
planning. Therefore, it is preferable to use the plan

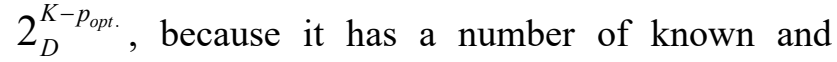
significant advantages over saturated planning [6], [7].

The variable is also involved in the planning of the extreme experiment as input data $I_{\text {full }}$, which determines the allowable excess of the number of experiments above the level of $K+1$ (when $I_{\text {full }}=0$, we have the saturated plan).

Generating ratios are given by the matrix $\left(\left(g_{r s}\right)\right) ; r \in \overline{1, p_{\text {opt. }}} ; s \in \overline{1, K_{\text {indep. }}}$. Here the value is the following: $K_{\text {indep. }}=K-p_{\text {opt. }}$. This matrix is formed algorithmically. The block diagram of such generation is shown in the Figure 2; although in [8] there is another version of it. Considering the natural choice $p=p_{\text {opt. }}$, the completion of the procedure can only occur on the branch 2 . The elements of the matrix $\left(\left(g_{r s}\right)\right) ; r \in \overline{1, p_{\text {opt. }}} ; s \in \overline{1, K_{\text {indep }}}$ are zeros and ones ("0" and " 1 ").

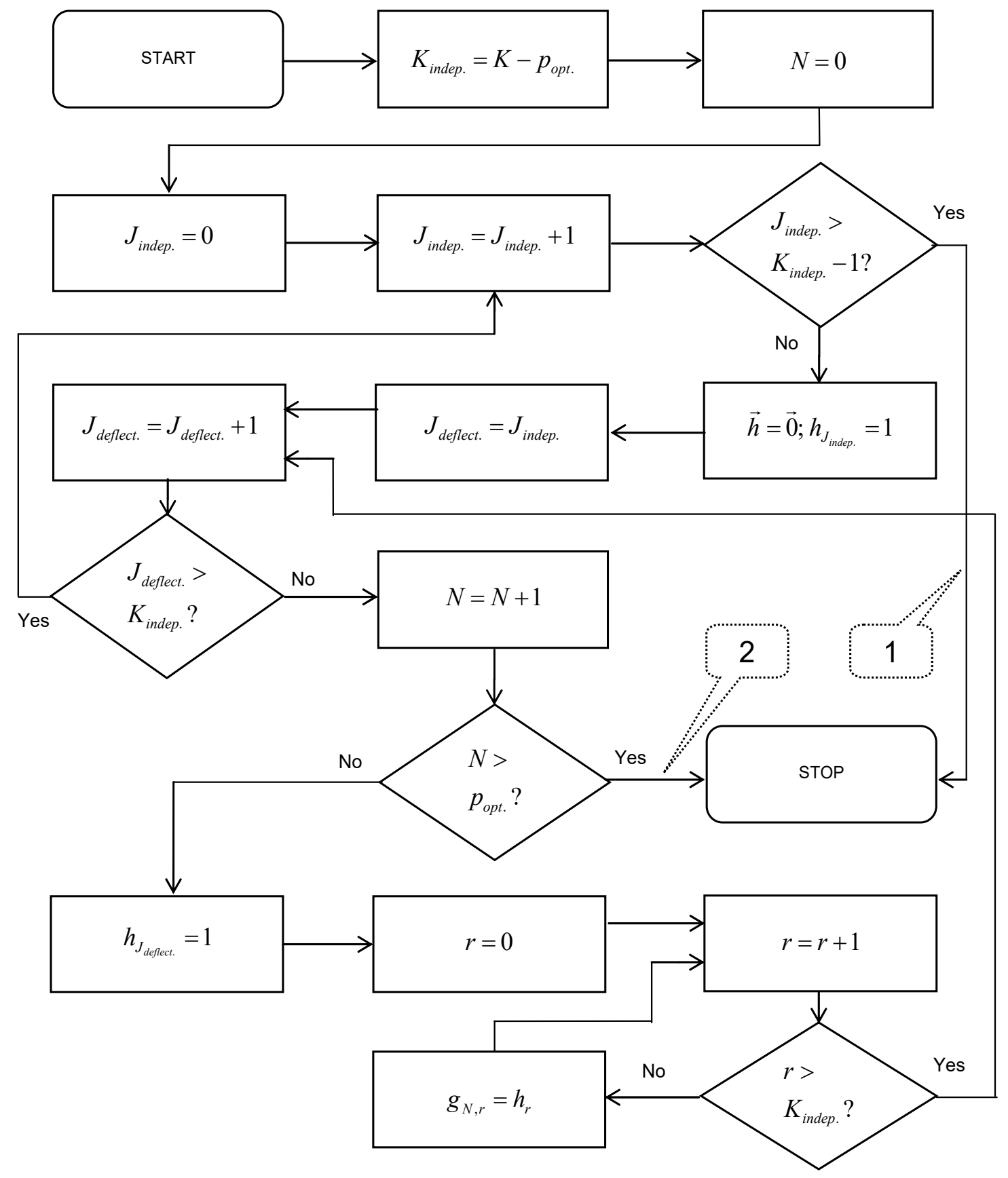

Figure 2. Block diagram of the algorithm for the synthesis of generating ratios 
To determine the elements of the experiment plan matrix "in levels" for independent variables (their total number is $N_{\text {exp. }}$ ) as the left sub-matrices of the matrix $\left(\left(g_{r s}\right)\right)$ for the first $K_{\text {indep. }}$ its columns we use its columns' empirically, here is the expression of the following type, which revealed the author:

$$
\left\{\begin{array}{l}
l_{m q}=(-1)^{G} ; m \in \overline{1, N_{\text {exp. }}} ; q \in \overline{1, K_{\text {indep. }}} ; \\
\left.G=\varepsilon\left[m+2^{q-1}-1\right) / 2^{q-1}\right]
\end{array}\right.
$$

where $\varepsilon(x)$ is the standard function taking the whole part of the argument $x$.

This expression is convenient, because it is analytical and does not require the implementation of an algorithmic procedure. Its finding seems to be an unexpected success of author.

Elements of the matrix of the experimental plan "in levels" as the right submatrix $\left(\left(g_{r s}\right)\right)$ for dependent variables for subsequent $K-K_{\text {indep. }}$ columns defined as follows:

$$
\begin{aligned}
& l_{m q}=\prod_{\lambda: g_{q \lambda}=1} l_{m \lambda} ; \\
& m \in \overline{1, N_{\text {exp. }}} ; q \in \overline{K_{\text {indep. }}+1, K} ; \lambda \in \overline{1, K_{\text {indep. }} .}
\end{aligned}
$$

The plan of the experiment in "natural values" is obtained from the plan of the experiment "in levels" as follows:

$$
X_{m i}=X_{i}^{0}+\Delta_{\text {min }_{i}} l_{m i} ; m \in \overline{1, N_{\text {exp. }}} ; i \in \overline{1, K},
$$

where $X_{i}^{0}$ is the entry-level of the factor $i$;

$\Delta_{\text {min }_{i}}$ is the quantization interval of the factor $i$, and it is obvious, that $\Delta_{\min _{i}}>0$.

After the carrying out experiments on the object in points $\left\{X_{m i}\right\} ; m \in \overline{1, N_{\text {exp. }}} ; i \in \overline{1, K}$ the matrix $\left(\left(\left(\left(X_{m i}\right)\right), y_{m}\right)\right)$, where $y_{m}$ is the response (result) for the experiment number $m$, that is in the point $m$ of the factor space $\left\{X_{m 1}, \ldots, X_{m K}\right\}$ is used for the LRM construction on the basis of the traditional formation of the system of normal equations and their solutions, for example, by Gauss-Jordan method.

If the planning of the experiment is saturated or an attempt to move along an inadequate LRM is allowed (sometimes this movement is appropriate), the transition to the movement along the LRM is carried out. Otherwise, the adequacy of the LRM is checked on the basis of traditional regression analysis $F$ statistics, in which the estimation of sample response variance is based on the results of additional $N_{c e n t}$. experiment in the center of the built plan to avoid the amendments by Mendenhall. It is advisable to recognize the following: $N_{\text {cent }}=5 ; \alpha=0,05$.

The movement along the LRM is need to find at each next step the coordinates of a new point $X_{i}^{\text {pres. }}$ in factor space in the following form:

$$
X_{i}^{\text {pres. }}=X_{i}^{\text {past }}+Q \Theta_{i} \Delta_{\min _{i}} ; i \in \overline{1, K},
$$

where $X_{i}^{\text {past }}$ are the coordinates of the previous point of the factor $i$;

$\Theta_{i}$ are the LRM performance coefficients of response surface;

$Q$ is one positive value that determines the length of the step when moving along the LRM in the direction of the optimum.

When the value $X_{i}^{\text {past }}$ is out of the bounding range of $\left[X_{i}^{\min }, X_{i}^{\max }\right]$, it is assumed to be equal to the corresponding boundary value that is reached.

Movement along the LRM stops is possible in either of two cases:

- if one has exhausted the allotted limit of experiments;

- if the sample at the new point is smaller or slightly larger than the previous one, as described above for the comparison algorithm based on technique of multiple comparisons.

\section{Procedure of investigation of Almost Stationary Area}

This procedure consists of iterations $I_{\text {plan }}$ (it is obvious, that $I_{\text {plan }} \geq 2$ ), each $j$-th is done the next operations.

1) The "star" plan is under construction for the factor $i$ with a length of "star" of the shoulder, which is equal to $2^{I_{\text {plan }}-j} \Delta_{\min _{i}}$ and the center in the optimum $X_{i}^{\text {opt. }}$, which was found in the previous iteration (for the case $j=1$ is the coordinate of the end point when there is a movement on the stage of steep ascent):

$$
X_{j}=X_{j}^{\text {opt. }} \pm 2^{I_{\text {plan }}-j} \Delta_{\text {min }_{i}} ; i \in \overline{1, K} .
$$

So, we get:

- For the first experiment:

$$
X_{1}^{\text {opt. }}+2^{I_{\text {plan }}-j} \Delta_{\text {min }_{i}}, X_{2}^{\text {opt. }}, \ldots, X_{K}^{\text {opt. }} ;
$$


- for the second experiment:

$$
X_{1}^{\text {opt. }}-2^{I_{\text {plan }}-j} \Delta_{\min _{i}}, X_{2}^{\text {opt. }}, \ldots, X_{K}^{\text {opt. }}
$$

- for the experiment number $2(K-1)$ :

$$
X_{1}^{\text {opt. }}, X_{2}^{\text {opt. }}, \ldots, X_{K}^{\text {opt. }}+2^{I_{\text {plan }}-j} \Delta_{\min _{K}}
$$

- for the experiment number $2 K$ :

$$
X_{1}^{\text {opt. }}, X_{2}^{\text {opt. }}, \ldots, X_{K}^{\text {opt. }}-2^{I_{\text {plan }}-j} \Delta_{\min _{K}} .
$$

The situation $X_{i} \notin\left[X_{i}^{\min }, X_{i}^{\max }\right]$ permitted it in the same way as it was described above.

2) When the inequality $K>K_{a d d}$. is satisfied (here $K_{\text {add. }}$ is one of priori boundary value, determined on the basis of the permissible number recalculation of the experiments, the limit number of variable factors), the realization of plan randomization on the basis of algorithm of a random sample from a set is organized [6], unlike [4], where random selection of factors and their levels was considered. This choice was due to the increase of homogeneity of the studied space "factors-response". This is also explained by the convenience of building algorithms and, accordingly, software implementation. The number of selected points of the factor space, in which the experiments with the object should be carried out is $N_{c h}=2 \min \left\{K, K_{\text {add. }}\right\}$.

3) For each of $N_{c h}$. points of the plan of the experiment under consideration, $d$ experiments with the object conduct.

4) From $N_{c h}+1$ samples of the realizations response (including the central one) with the length $d$ each one, it is chosen the length with a significantly better (larger) sample mean, and it becomes the center of the next "star" plan (if $j<I_{\text {plan. }}$ ) or it is considered a rational result of the experiment (if $j=I_{\text {plan. }}$ ).

5) We complete the line protocol of investigation of almost stationary region (trajectory protocol):

$$
W_{j i}=\hat{X}_{i} ; i \in \overline{1, K},
$$

where $\hat{X}_{i}$ are the coordinates of the point corresponding to the significantly better sample response mean for the given iteration of the factor $i$.

The total number of experiments at the research stage of almost stationary region is:

$$
N_{\text {res.s.s.a. }}=2 I_{\text {plan }} d \min \left\{K, K_{\text {add. }}\right\} .
$$

\section{Procedure for evaluating of the Remoteness of the Obtained Rational Solution by Comparison with the Unknown Optimal One}

This procedure is based on the analysis of the trajectory protocol formed at the previous stage.

The boundaries $\left\{b_{i 1}, b_{i 2}\right\}$ are placed in series for each factor $i$ as follows.

If:

- the starting point was the best one

$$
\left\{\begin{array}{l}
b_{i 1}=W_{i 1}-\Delta_{\min _{i}} ; \\
b_{i 2}=W_{i 1}+\Delta_{\min _{i}}
\end{array} ;\right.
$$

- the movement took place only to the left

$$
\left\{\begin{array}{l}
b_{i 1}=X_{i}^{\mathrm{min}} \\
b_{i 2}=X_{i}^{0}
\end{array}\right.
$$

- the movement took place only to the right

$$
\left\{\begin{array}{l}
b_{i 1}=X_{i}^{0} \\
b_{i 2}=X_{i}^{\max }
\end{array}\right.
$$

- the direction of motion was alternated

$$
\left\{\begin{array}{l}
b_{i 1}-\text { is the nearest to the } W_{I_{\text {plan }}, i} \text { left } \\
\text { from the value of }\left\{W_{g i}\right\} ; \\
b_{i 2}-\text { is the nearest to the } W_{I_{p l a n}, i} \text { right } \\
\text { from the value of }\left\{W_{g i}\right\},
\end{array}\right.
$$

where $X_{i}^{0}$ are the coordinates of the starting point; $\left[X_{i}^{\min }, X_{i}^{\max }\right]$ is the allowable range of variation of the factor $X_{i}$.

\section{The Software Implementation of the Algorithm}

The algorithm described above was implemented in ForTran language. Its software implementation is applicable in almost all available ForTran environments, starting with ForTran-IV if ignoring the problem of the interactive shell. A software implementation is autonomous. There are no problems with its migration to other language environments, as it seems for author. The program has a block-modular structure (see Figure 3 ) with the allocation of subroutine type "SUBROUTINE" and within a wide range is invariant with respect to the values of the initial data. Anyone can set arbitrary (of 
course, common) values $K, I_{\text {full }}, Q, I_{\text {plan }}, D,\left\{X_{i}^{0}\right\},\left\{X_{i}^{\min }\right\},\left\{X_{i}^{\max }\right\}$, $\left\{\Delta_{\min _{i}}\right\}$ and algorithm parameter $\alpha$. At the change

of the algorithm parameters $d$ and $K_{a d d}$, only need to change the dimensions of the corresponding arrays.

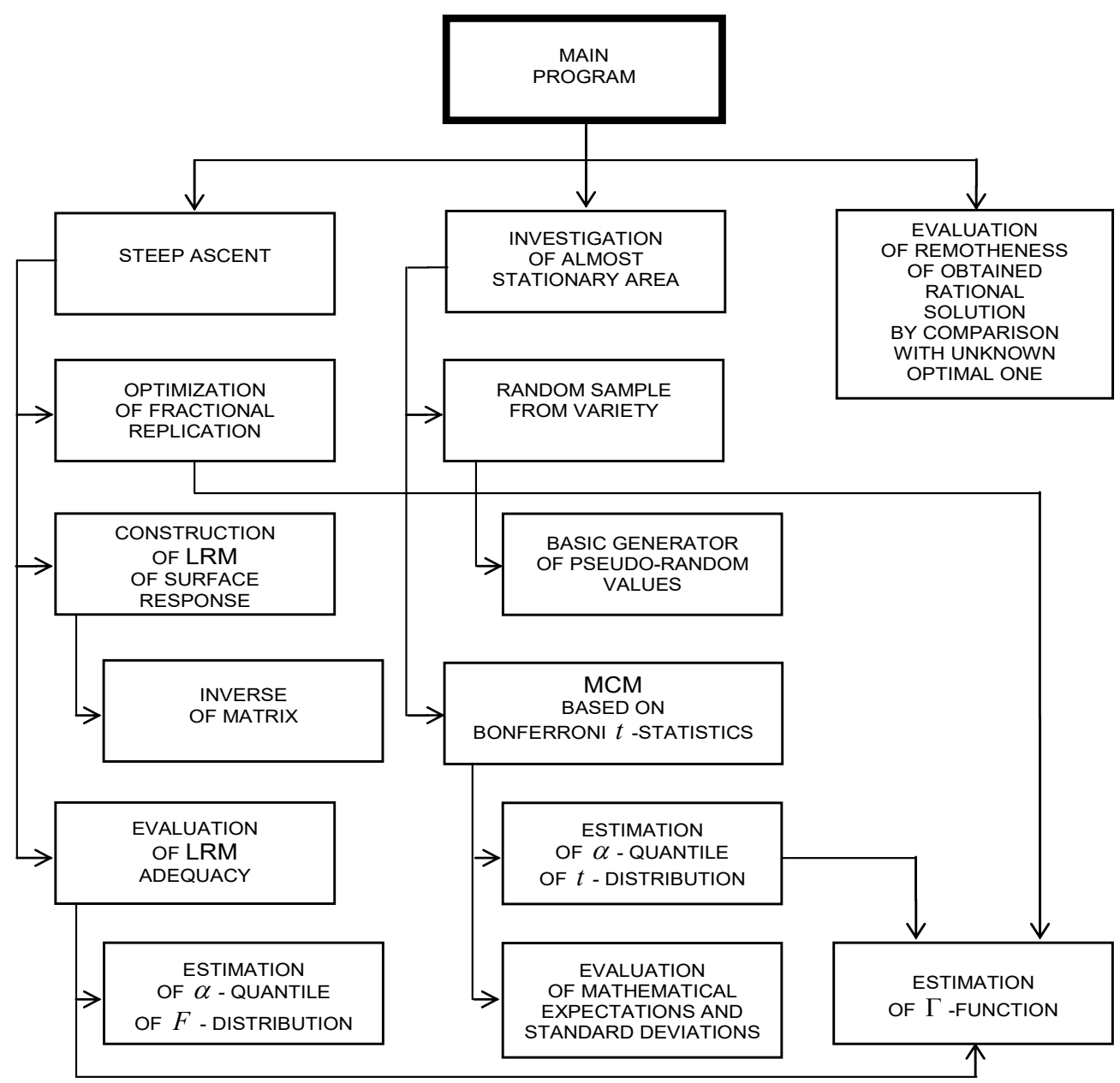

Figure 3. Structure of software implementation of the algorithm

The program includes 17 subroutines with the number of program lines executed (source statements) equal to 716 .

It is carried out in practice almost in real time.

In particular, the software implementation matched with implementations of "heavy" simulation models. At the same time, the level of nesting of the subroutines reached the level 20 i.e. went beyond the level that has not been investigated even by many developers of specialized software environments.

Note that in the course of algorithmization, the procedure for accounting voluntarily assigned points of factor space was not formalized. They were simply introduced as additional in the investigation of an almost stationary region.

Also, it was not possible to cope radically with the conceptual problem of optimization: the distinction between local and global optima.

\section{Introduction of the Developed Algorithm and its Software Implementation}

The developed algorithm has been successfully applied in general in the following areas, in particular:

- when optimizing production programs in physical terms, increases reliability (above all reliability and durability) and fleet prices of a significant number of types of aircraft and their components, including aircraft engines, in terms of ensuring the implementation of the final aviation work for various purposes. Along with this, the transfer of some positions of aviation technology to the so-called progressive strategies of technical operation was optimized. The total number of computer experiments planned and conducted in the period 1983-1992 amounted to several hundred;

- while optimizing the programs of introducing some advanced materials into the structural performance of aircraft in the period 1983-1992; 
- during the formation of the pricing system for the results of carrying out pile-testing and re-static tests of certain types of aircraft in the second half of the 1980s;

- when optimizing the operating conditions of a number of high-tech enterprises and corporate groupings of a holding type in Russia in the period 1996-2002, in terms of the fiscal nature of the tax system, investment load, etc.;

- while optimizing the programs of technical reequipment of a number of high-tech domestic productions in terms of the type and number of high-tech technological equipment in the period 1988-2002;

- when performing a number of feasibility studies of important institutional, investment and innovation projects in terms of generating operating scenarios and implementation alternatives in the period 1988-2018.

\section{Conclusions}

Thus, in the course of the study, prerequisites were created in order to reasonably formulate the following findings, conclusions and recommendations:

- the problem of planning an extreme experiment is relevant in relation to objects, especially for informationally complex objects, of a technical, economic and informational nature;

- the planning algorithms for such experiments are not subject to large-scale and in-depth research, which significantly reduces the quality of management;

- the number of severe restrictions are imposed on the algorithm for planning an extreme experiment, primarily due to the high resource intensity of the experiments;

- in some cases, it is advisable to unify such algorithms, extending their application to cases that include technical, economic, information and other objects, as well as their models: natural, semi-natural and mathematical (analytical and simulation);

- the number of stringent requirements are imposed on unified algorithms for planning an extreme experiment;

- it is appropriate to segment a unified algorithm and implement a quick exit to the region of contiguity to the optimum and its careful study and evaluation of distance from an unknown optimum;

- with the forming a unified algorithm of an extreme experiment there are a lot of design mathematical optimization problems, examples of solutions which show the productivity of abandoning the production concept according to the "as is" scheme (as it turned out);

- the application of the development of the considered type is feasible and productive.

\section{References}

[1]. Bazadze, N. G., (2005). Organizacionnoe proektirovaniye i razvitiye [Organizational design and development]. Moscow: Dobroe slovo.

[2]. Dmitriev, O. N., Ekshembiev, S. K., \& Lyubaeva, Z. I. (2013). Strategicheskoe upravlenie korporatsiei (fundamental'nye i prikladnye problemy).

[3]. Demchenko, O. F. (2011). Metodologiya matematicheskogo modelirovaniya organizatsionnykh struktur aviatsionno-promyshlennogo kompleksa Rossiiskoi Federatsii (Methodology of mathematical modeling of organizational structures for aviation industrial complex of the Russian Federation). Moscow, KnoRus.

[4]. Kleijnen, J. P. (1978). The role of statistical methodology in simulation. ACM SIGSIM Simulation Digest, 10(1-2), 51-52.

[5]. Kleijnen, J. P. (2015, September). Design and analysis of simulation experiments. In International Workshop on Simulation (pp. 3-22). Springer, Cham.

[6]. Knuth, D. E. (1976). The state of the Art of Computer Programming (No. STAN-CS-76-551). Stanford Univ Ca Dept Of Computer Science.

[7]. Nalimov, V. V., Golihova, T. I., \& Mikeshina, N. G. (1970). On practical use of the concept of Doptimality. Technometrics, 12(4), 799-812.

[8]. Dmitriev, O. N. (2002). Intellektual'naya informatsionnaya tekhnologiya tekhnikoekonomicheskogo obosnovaniya marketingovykh reshenii (Intelligent Information Technology for Feasibility Study Marketing Solutions). Moscow: Gnom i D.

[9]. Fang, K. T., Li, R., \& Sudjianto, A. (2005). Design and modeling for computer experiments. CRC press.

[10]. F. Pukelsheim. (2006). Optimal Design of Experiments, Philadelphia, PA, USA:SIAM.

[11]. Antony, J. (2014). Design of experiments for engineers and scientists. Elsevier.

[12]. Aveiro, P. (2016). Design of experiments in production engineering. J. P. Davim (Ed.). Basel, Switzerland:: Springer International Publishing.

[13]. Giesbrecht, F. G., \& Gumpertz, M. L. (2011). Planning, construction, and statistical analysis of comparative experiments (Vol. 511). John Wiley \& Sons.

[14]. Shmelova, T., Sikirda, Y., Rizun, N., Kucherov, D., \& Dergachov, K. (Eds.). (2019). Automated Systems in the Aviation and Aerospace Industries. IGI Global.

[15]. Dahan, E., \& Mendelson, H. (2001). An extremevalue model of concept testing. Management science, 47(1), 102-116.

[16]. Park, S., Fowler, J. W., Mackulak, G. T., Keats, J. B., \& Carlyle, W. M. (2002). D-optimal sequential experiments for generating a simulation-based cycle time-throughput curve. Operations research, 50(6), 981-990.

[17]. Przybylski, A., Gandibleux, X., \& Ehrgott, M. (2010). A recursive algorithm for finding all nondominated extreme points in the outcome set of a multiobjective integer programme. INFORMS Journal on Computing, 22(3), 371-386. 